\title{
Consistency of the Adiabatic Theorem
}

\author{
M.S. Sarandy, L.-A. Wu, D.A. Lidar \\ Chemical Physics Theory Group, Department of Chemistry, \\ and Center for Quantum Information and Quantum Control, \\ University of Toronto, 80 St. George Street, Toronto, Ontario, M5S 3H6, Canada
}

\begin{abstract}
We review the quantum adiabatic approximation for closed systems, and its recently introduced generalization to open systems (M.S. Sarandy and D.A. Lidar, e-print quant-ph/0404147). We also critically examine a recent argument claiming that there is an inconsistency in the adiabatic theorem for closed quantum systems [K.P. Marzlin and B.C. Sanders, Phys. Rev. Lett. 93, 160408 (2004)] and point out how an incorrect manipulation of the adiabatic theorem may lead one to obtain such an inconsistent result.
\end{abstract}

PACS numbers: 03.65.Ta, 03.65.Yz, 03.67.-a, 03.65.Vf

Keywords: Adiabatic Theorem; Berry's Phases; Open Quantum Systems; Quantum Computation.

\section{INTRODUCTION}

The adiabatic theorem [1, 2, 3, 4] is one of the oldest and most widely used general tools in quantum mechanics. The theorem concerns the evolution of systems subject to slowly varying Hamiltonians. Roughly, its content is that if a state is an instantaneous eigenstate of a sufficiently slowly varying $H$ at one time then it will remain an eigenstate at later times, while its eigenenergy evolves continuously. When the slowness assumption is relaxed transitions become weakly allowed [5, 6, 7, [8]. The role of the adiabatic theorem in the study of slowly varying quantum mechanical systems spans a vast array of fields and applications, such as the Landau-Zener theory of energy level crossings in molecules [9, 10], quantum field theory [1], and Berry's phase 12]. In recent years geometric phases [13] have been proposed to perform quantum information processing 14, 15, 16], with adiabaticity assumed in a number of schemes for geometric quantum computation (e.g., 17, 18, 19, 20]). Additional interest in adiabatic processes has arisen in connection with the concept of adiabatic quantum computing, in which slowly varying Hamiltonians appear as a promising mechanism for the design of new quantum algorithms and even as an alternative to the conventional quantum circuit model of quantum computation [21, 22, 23].

More recently, in Ref. [24], the adiabatic theorem was generalized to the case of open quantum systems, i.e., quantum systems coupled to an external environment. Instead of making use of eigenstates of the Hamiltonian, adiabaticity is defined through the Jordan canonical form of the generator of the master equation governing the dynamics of the system. This new framework allowed for the derivation of an adiabatic approximation which includes the case of systems evolving in the presence of noise. This issue is particularly important in the context of quantum information processing, where environment induced decoherence is viewed as a fundamental obstacle on the path to the construction of quantum computers (e.g., 25]).

The aim of this paper is to review the adiabatic approximation in quantum mechanics for both closed and open quantum systems as well as to point out how an incorrect manipulation of the adiabatic theorem can yield an inconsistent result. Indeed, in a recent paper entitled "Inconsistency in the application of the adiabatic theorem" 26] the authors argue that there may be an inconsistency in the adiabatic theorem for closed quantum systems. We show here how this inconsistency can be resolved. Related discussions can be found in Refs. [27, 28, 29].

\section{THE QUANTUM ADIABATIC APPROXIMATION FOR CLOSED SYSTEMS}

\section{A. Condition on the Hamiltonian}

Let us begin by reviewing the adiabatic approximation in closed quantum systems, which evolve unitarily through a time-dependent Schrödinger equation

$$
H(t)|\psi(t)\rangle=i|\dot{\psi}(t)\rangle
$$

where $H(t)$ denotes the Hamiltonian and $|\psi(t)\rangle$ is a quantum state in a $D$-dimensional Hilbert space. We use units where $\hbar=1$. For simplicity we assume that the spectrum of $H(t)$ is entirely discrete and nondegenerate. Thus we can define an instantaneous basis of eigenenergies by

$$
H(t)|n(t)\rangle=E_{n}(t)|n(t)\rangle,
$$


with the set of eigenvectors $|n(t)\rangle$ chosen to be orthonormal. In this simplest case, where to each energy level there corresponds a unique eigenstate we can define adiabaticity as the regime associated to an independent evolution of the instantaneous eigenvectors of $H(t)$.

This means that instantaneous eigenstates at one time evolve continuously to the corresponding eigenstates at later times, and that their corresponding eigenenergies do not cross. In particular, if the system begins its evolution in a particular eigenstate $|n(0)\rangle$ then it will evolve to the instantaneous eigenstate $|n(t)\rangle$ at a later time $t$, without any transition to other energy levels.

It is conceptually useful to point out that the relationship between slowly varying Hamiltonians and adiabatic behavior can be demonstrated directly from a simple manipulation of the Schrödinger equation: recall that $H(t)$ can be diagonalized by a unitary similarity transformation

$$
H_{d}(t)=U^{-1}(t) H(t) U(t)
$$

where $H_{d}(t)$ denotes the diagonalized Hamiltonian and $U(t)$ is a unitary transformation. Multiplying Eq. (1) by $U^{-1}(t)$ and using Eq. (3) we obtain

$$
H_{d}|\psi\rangle_{d}=i|\dot{\psi}\rangle_{d}-i \dot{U}^{-1}|\psi\rangle
$$

where $|\psi\rangle_{d} \equiv U^{-1}|\psi\rangle$ is the state of the system in the basis of eigenvectors of $H(t)$. Upon considering that $H(t)$ changes slowly in time, i.e. $d H(t) / d t \approx 0$, we may also assume that the unitary transformation $U(t)$ and its inverse $U^{-1}(t)$ are slowly varying operators, yielding

$$
H_{d}(t)|\psi(t)\rangle_{d}=i|\dot{\psi}(t)\rangle_{d}
$$

Thus, since $H_{d}(t)$ is diagonal, the system evolves separately in each energy sector, ensuring the validity of the adiabatic approximation.

Now let

$$
g_{n k}(t) \equiv E_{n}(t)-E_{k}(t)
$$

be the energy gap between level $n$ and $k$ and let $T$ be the total evolution time. One may then state $a$ general validity condition for adiabatic behavior as follows:

$$
\max _{0 \leq t \leq T}\left|\frac{\langle k|\dot{H}| n\rangle}{g_{n k}}\right| \ll \min _{0 \leq t \leq T}\left|g_{n k}\right| .
$$

Note that the left-hand side of Eq. (7) has dimensions of frequency and hence must compared to the relevant physical frequency scale, which can be proved to be given by the gap $g_{n k}$ [4, 30]. [In fact, Eq. (7) will be seen to be a direct consequence of the adiabatic condition derived in Subsection [IB] The interpretation of the adiabaticity condition (77) is that for all pairs of energy levels, the expectation value of the time-rate-of-change of the Hamiltonian, in units of the gap, must be small compared to the gap. For a discussion of the adiabatic regime when there is no gap in the energy spectrum see Ref. [31].

In order to obtain Eq. (7), let us expand $|\psi(t)\rangle$ in terms of the basis of instantaneous eigenvectors of $H(t)$ :

$$
|\psi(t)\rangle=\sum_{n=1}^{D} a_{n}(t) e^{-i \int_{0}^{t} d t^{\prime} E_{n}\left(t^{\prime}\right)}|n(t)\rangle,
$$

with $a_{n}(t)$ being complex functions of time. Substitution of Eq. (8) into Eq. (11) and multiplying the result by $\langle k(t)|$, we have

$$
\dot{a}_{k}=-\sum_{n} a_{n}\langle k \mid \dot{n}\rangle e^{-i \int_{0}^{t} d t^{\prime} g_{n k}\left(t^{\prime}\right)} .
$$

A useful expression for $\langle k \mid \dot{n}\rangle$, for $k \neq n$, can be found by taking a time derivative of Eq. (2) and multiplying the resulting expression by $\langle k|$, which reads

$$
\langle k \mid \dot{n}\rangle=\frac{\langle k|\dot{H}| n\rangle}{g_{n k}} \quad(n \neq k) .
$$


Therefore Eq. (9) can be written as

$$
\dot{a}_{k}=-a_{k}\langle k \mid \dot{k}\rangle-\sum_{n \neq k} a_{n} \frac{\langle k|\dot{H}| n\rangle}{g_{n k}} e^{-i \int_{0}^{t} d t^{\prime} g_{n k}\left(t^{\prime}\right)} .
$$

Adiabatic evolution is ensured if the coefficients $a_{k}(t)$ evolve independently from each other, i.e., if their dynamical equations do not couple. As is apparent from Eq. (11), this requirement is fulfilled when the condition (7) is imposed.

In the case of a degenerate spectrum of $H(t)$, Eq. (10) holds only for eigenstates $|k\rangle$ and $|n\rangle$ for which $E_{n} \neq E_{k}$. Taking into account this modification in Eq. (11), it is not difficult to see that the adiabatic approximation generalizes to the statement that each degenerate eigenspace of $H(t)$, instead of individual eigenvectors, has independent evolution, whose validity conditions given by Eq. (7) are to be considered over eigenvectors with distinct energies. Thus, in general one can define adiabatic dynamics of closed quantum systems as follows:

Definition II.1 A closed quantum system is said to undergo adiabatic dynamics if its Hilbert space can be decomposed into decoupled Schrödinger-eigenspaces with distinct, time-continuous, and non-crossing instantaneous eigenvalues of $H(t)$.

\section{B. Condition on the total evolution time}

A very useful alternative is to express the adiabaticity condition in terms of the total evolution time $T$. We shall consider for simplicity a nondegenerate $H(t)$; the generalization to the degenerate case is also possible. Taking the initial state as the eigenvector $|m(0)\rangle$, with $a_{m}(0)=1$, the condition for adiabatic evolution can be stated as follows:

$$
T \gg \frac{\mathcal{F}}{\mathcal{G}^{2}}
$$

where

$$
\mathcal{F}=\max _{0 \leq s \leq 1}\left|\left\langle k(s)\left|\frac{d H(s)}{d s}\right| m(s)\right\rangle\right|, \quad \mathcal{G}=\min _{0 \leq s \leq 1}\left|g_{m k}(s)\right| .
$$

Eq. (12) can be interpreted as stating the total evolution time must be much larger than the norm of the timederivative of the Hamiltonian divided by the square of the energy gap. It gives an important validity condition for the adiabatic approximation, which has been used, e.g., to determine the running time required by adiabatic quantum algorithms [21, 22, 23]. By using the time variable transformation (16), one can show that Eq. (12) is indeed equivalent to the adiabatic condition (7) on the Hamiltonian.

To derive Eq. (12), let us rewrite Eq. (11) as follows [32]:

$$
e^{i \gamma_{k}(t)} \frac{\partial}{\partial t}\left(a_{k}(t) e^{-i \gamma_{k}(t)}\right)=-\sum_{n \neq k} a_{n} \frac{\langle k|\dot{H}| n\rangle}{g_{n k}} e^{-i \int_{0}^{t} d t^{\prime} g_{n k}\left(t^{\prime}\right)}
$$

where $\gamma_{k}(t)$ denotes the Berry's phase [12] associated to the state $|k\rangle$ :

$$
\gamma_{k}(t)=i \int_{0}^{t} d t^{\prime}\left\langle k\left(t^{\prime}\right) \mid \dot{k}\left(t^{\prime}\right)\right\rangle
$$

Now let us define a normalized time $s$ through the variable transformation

$$
t=s T, \quad 0 \leq s \leq 1
$$

Then, by performing the change $t \rightarrow s$ in Eq. (14) and integrating we obtain

$$
a_{k}(s) e^{-i \gamma_{k}(s)}=a_{k}(0)-\sum_{n \neq k} \int_{0}^{s} d s^{\prime} \frac{F_{n k}\left(s^{\prime}\right)}{g_{n k}\left(s^{\prime}\right)} e^{-i T \int_{0}^{s^{\prime}} d s^{\prime \prime} g_{n k}\left(s^{\prime \prime}\right)}
$$

where

$$
F_{n k}(s)=a_{n}(s)\left\langle k(s)\left|\frac{d H(s)}{d s}\right| n(s)\right\rangle e^{-i \gamma_{k}(s)}
$$


However, for an adiabatic evolution as defined above, the coefficients $a_{n}(s)$ evolve without any mixing, which means that $a_{n}(s) \approx a_{n}(0) e^{i \gamma_{n}(s)}$. Therefore

$$
F_{n k}(s)=a_{n}(0)\left\langle k(s)\left|\frac{d H(s)}{d s}\right| n(s)\right\rangle e^{-i\left(\gamma_{k}(s)-\gamma_{n}(s)\right)} .
$$

In order to arrive at a condition on $T$ it is useful to separate out the fast oscillatory part from Eq. (17). Thus, the integrand in Eq. (17) can be rewritten as

$$
\frac{F_{n k}\left(s^{\prime}\right)}{g_{n k}\left(s^{\prime}\right)} e^{-i T \int_{0}^{s^{\prime}} d s^{\prime \prime} g_{n k}\left(s^{\prime \prime}\right)}=\frac{i}{T}\left[\frac{d}{d s^{\prime}}\left(\frac{F_{n k}\left(s^{\prime}\right)}{g_{n k}^{2}\left(s^{\prime}\right)} e^{-i T \int_{0}^{s^{\prime}} d s^{\prime \prime} g_{n k}\left(s^{\prime \prime}\right)}\right)-e^{-i T \int_{0}^{s^{\prime}} d s^{\prime \prime} g_{n k}\left(s^{\prime \prime}\right)} \frac{d}{d s^{\prime}}\left(\frac{F_{n k}\left(s^{\prime}\right)}{g_{n k}^{2}\left(s^{\prime}\right)}\right)\right] .
$$

Substitution of Eq. (20) into Eq. (17) results in

$$
a_{k}(s) e^{-i \gamma_{k}(s)}=a_{k}(0)+\frac{i}{T} \sum_{n \neq k}\left(\frac{F_{n k}(0)}{g_{n k}^{2}(0)}-\frac{F_{n k}(s)}{g_{n k}^{2}(s)} e^{-i T \int_{0}^{s} d s^{\prime} g_{n k}\left(s^{\prime}\right)}+\int_{0}^{s} d s^{\prime} e^{-i T \int_{0}^{s^{\prime}} d s^{\prime \prime} g_{n k}\left(s^{\prime \prime}\right)} \frac{d}{d s^{\prime}} \frac{F_{n k}\left(s^{\prime}\right)}{g_{n k}^{2}\left(s^{\prime}\right)}\right)
$$

A condition for the adiabatic regime can be obtained from Eq. (21) if the last integral vanishes for large T. Let us assume that, as $T \rightarrow \infty$, the energy difference remains nonvanishing. We further assume that $d\left\{F_{n k}\left(s^{\prime}\right) / g_{n k}^{2}\left(s^{\prime}\right)\right\} / d s^{\prime}$ is integrable on the interval $[0, s]$. Then it follows from the Riemann-Lebesgue lemma 33] that the last integral in Eq. (21) vanishes in the limit $T \rightarrow \infty$ (due to the fast oscillation of the integrand) [34]. What is left are therefore only the first two terms in the sum over $n \neq k$ of Eq. (21). Thus, a general estimate of the time rate at which the adiabatic regime is approached can be expressed by

$$
T \gg \frac{F}{g^{2}}
$$

where

$$
F=\max _{0 \leq s \leq 1}\left|a_{n}(0)\left\langle k(s)\left|\frac{d H(s)}{d s}\right| n(s)\right\rangle\right|, \quad g=\min _{0 \leq s \leq 1}\left|g_{n k}(s)\right|
$$

with max and min taken over all $k$ and $n$. Eq. (12) is then obtained as the special case when the system starts its evolution in a particular eigenstate of $H(t)$.

\section{Higher-order corrections to the adiabatic approximation}

When the Hamiltonian of a quantum system changes slowly, but not extremely slowly, the degenerate eigenspaces of $H(t)$ (or individual eigenvectors in the case of nondegenerate spectrum) will not evolve completely independently from each other and, therefore, the dynamical equation (9) will weakly couple distinct eigenspaces of $H(t)$. Then, for non-extremely slowly varying Hamiltonians, the adiabatic solution is actually a zeroth-order approximation and higherorder corrections must be considered. Some higher-order adiabatic approximation methods have been proposed 5 , 6, 7, 8]. Here we shall review, for the non-degenerate case, the method proposed by Wu in Ref. 8]. Let us begin by expanding the state vector $|\psi(t)\rangle$ in the instantaneous eigenbasis, as in Eq. (8). Then, by using the normalized time $s$ introduced in Eq. (16) we obtain the following matrix form for the Schrödinger equation

$$
\frac{d \psi(s)}{d s}=K(s) \psi(s)
$$

where $K(s)$ is an anti-Hermitian matrix with elements

$$
K_{m n}(s)=-\left\langle m(s)\left|\frac{d}{d s}\right| n(s)\right\rangle \exp \left(i T \int_{0}^{s} d s^{\prime} g_{m n}\left(s^{\prime}\right)\right) .
$$

The matrix $K(s)$ can be separated into a diagonal matrix $D(s)$ and an off-diagonal matrix $O(s)$, yielding

$$
K(s)=D(s)+O(s) .
$$

The evolution operator $U(s)$ for the system satisfies the equation

$$
\frac{d U(s)}{d s}=K(s) U(s), \quad(\text { with } U(0)=1)
$$


which, after integration and use of Eq. (26), becomes

$$
U(s)=1+\int_{0}^{s} d s_{1}\left[D\left(s_{1}\right)+O\left(s_{1}\right)\right]+\int_{0}^{s} d s_{1} \int_{0}^{s_{1}} d s_{2}\left[D\left(s_{1}\right)+O\left(s_{1}\right)\right]\left[D\left(s_{2}\right)+O\left(s_{2}\right)\right]+\ldots
$$

Now let us define

$$
U^{(0)}(s)=1+\int_{0}^{s} d s_{1} D\left(s_{1}\right)+\int_{0}^{s} d s_{1} \int_{0}^{s_{1}} d s_{2} D\left(s_{1}\right) D\left(s_{2}\right)+\ldots,
$$

which involves only the diagonal parts. Moreover, for $n>0$, we denote $U^{(n)}(s)$ as the sum of all the integrals with $n$ off-diagonal $O(s)$ factors in the integrand. Therefore the evolution operator can be expanded in powers of the off-diagonal matrices $O(s)$ as $U(s)=\sum_{n=0} U^{(n)}(s)$. It can be shown [8] that the $n^{\text {th }}$ term $U^{(n)}(s)$ can be expressed through the lower order term $U^{(n-1)}(s)$ by means of the recurrence equation

$$
U^{(n)}(s)=\int_{0}^{s} d s^{\prime} U^{(0)}\left(s^{\prime}\right) O\left(s^{\prime}\right) U^{(n-1)}\left(s^{\prime}\right) .
$$

The expression above means that, by knowing the zeroth-order evolution operator $U^{(0)}(s)$, which exactly yields the adiabatic approximation, one can obtain $U^{(1)}(s)$, and then $U^{(2)}(s)$ and so on. The adiabatic case corresponds to no transitions, while a correction $U^{(n)}(s)$ of order $n \geq 1$ implies the existence of $n$ transitions between different energy levels [8]. From this perspective one can interpret the adiabatic approximation as the zeroth order term in a perturbation theory in the number of transitions between energy levels connected by the time-varying Hamiltonian.

\section{THE QUANTUM ADIABATIC APPROXIMATION FOR OPEN QUANTUM SYSTEMS}

In this section we review our recently introduced generalization of the adiabatic theorem to the case of open quantum systems 24]. The motivations for considering such a generalization are many. The most fundamental is that the concept of a closed system is, of course, an idealization, and in reality all experimentally accessible systems are open. Thus applications of the adiabatic theorem for open systems include, among others, geometric phases (where open system effects have received considerable recent attention, e.g., Refs. [35]), quantum information processing, and molecular dynamics in condensed phases.

In the following we first introduce notation for open systems, then discuss the generalized adiabatic theorem.

\section{A. The dynamics of open quantum system}

Consider a quantum system $S$ coupled to an environment, or bath $B$ (with respective Hilbert spaces $\mathcal{H}_{S}, \mathcal{H}_{B}$ ), evolving unitarily under the total system-bath Hamiltonian $H_{S B}$. The exact system dynamics is given by tracing over the bath degrees of freedom 36

$$
\rho(t)=\operatorname{Tr}_{B}\left[U(t) \rho_{S B}(0) U^{\dagger}(t)\right]
$$

where $\rho(t)$ is the system state, $\rho_{S B}(0)=\rho(0) \otimes \rho_{B}(0)$ is the initially uncorrelated system-bath state, and $U(t)=$ $\mathcal{T} \exp \left(-i \int_{0}^{t} H_{S B}\left(t^{\prime}\right) d t^{\prime}\right)(\mathcal{T}$ denotes time-ordering). Such an evolution is completely positive and trace preserving 36, 37, 38]. Under certain approximations, it is possible to convert Eq. (31) into the convolutionless form

$$
\dot{\rho}(t)=\mathcal{L}(t) \rho(t) .
$$

An important example is

$$
\dot{\rho}(t)=-i[H(t), \rho(t)]+\frac{1}{2} \sum_{i=1}^{N}\left(\left[\Gamma_{i}(t), \rho(t) \Gamma_{i}^{\dagger}(t)\right]+\left[\Gamma_{i}(t) \rho(t), \Gamma_{i}^{\dagger}(t)\right]\right) .
$$

Here $H(t)$ is the time-dependent effective Hamiltonian of the open system and $\Gamma_{i}(t)$ are time-dependent operators describing the system-bath interaction. In the literature, Eq. (33) with time-independent operators $\Gamma_{i}$ is usually referred to as the Markovian dynamical semigroup, or Lindblad equation [36, 38, 39, 40] [see also Ref. [41] for a simple derivation of Eq. (33) from Eq. (31)]. However, the case with time-dependent coefficients is also permissible under certain restrictions [4]. The Lindblad equation requires the assumption of a Markovian bath with vanishing correlation 
time. Equation (32) can be more general; for example, it applies to the case of non-Markovian convolutionless master equations studied in Ref. [43]. Here we will consider the class of convolutionless master equations (32). In a slight abuse of nomenclature, we will henceforth refer to the time-dependent generator $\mathcal{L}(t)$ as the Lindblad superoperator, and the $\Gamma_{i}(t)$ as Lindblad operators.

Conceptually, the difficulty in the transition of an adiabatic approximation from closed to open quantum systems is that the notion of Hamiltonian eigenstates is lost, since the Lindblad superoperator - the generalization of the Hamiltonian - cannot in general be diagonalized. It is then not a priori clear what should take the place of the adiabatic eigenstates. This difficulty was solved in Ref. 24] by introducing the idea that this role is played by adiabatic Jordan blocks of the Lindblad superoperator. The Jordan canonical form [44], with its associated left- and right-eigenvectors, is in this context the natural generalization of the diagonalization of the Hamiltonian. In this direction, it is convenient to work in the superoperator formalism, wherein the density matrix is represented by a $D^{2}$-dimensional "coherence vector"

$$
|\rho\rangle\rangle=\left(\begin{array}{llll}
\rho_{1} & \rho_{2} & \cdots & \rho_{D^{2}}
\end{array}\right)^{t},
$$

and the Lindblad superoperator $\mathcal{L}$ becomes a $D^{2} \times D^{2}$-dimensional supermatrix [38]. We use the double bracket notation to indicate that we are not working in the standard Hilbert space of state vectors. More generally, coherence vectors live in Hilbert-Schmidt space: a state space of linear operators endowed with an inner product that can be defined, for general vectors $u$ and $v$, as

$$
(u, v) \equiv\langle\langle u \mid v\rangle\rangle \equiv \frac{1}{\mathcal{N}} \operatorname{Tr}\left(u^{\dagger} v\right) .
$$

where $\mathcal{N}$ is a normalization factor. Adjoint elements $\langle\langle v|$ in the dual state space are given by row vectors defined as the transpose conjugate of $|v\rangle\rangle:\left\langle\langle v|=\left(v_{1}^{*}, v_{2}^{*}, \ldots, v_{D^{2}}^{*}\right)\right.$. A density matrix can then be expressed as a discrete superposition of states over a complete basis in this vector space, with appropriate constraints on the coefficients so that the requirements of Hermiticity, positive semi-definiteness and unit trace of $\rho$ are observed. Thus, representing the density operator in general as a coherence vector, we can rewrite Eq. (32) in a superoperator language as

$$
\mathcal{L}(t)|\rho(t)\rangle\rangle=|\dot{\rho}(t)\rangle\rangle
$$

where $\mathcal{L}$ is now a supermatrix. This master equation generates non-unitary evolution, since $\mathcal{L}(t)$ is non-Hermitian and hence generally non-diagonalizable. However, one can always transform $\mathcal{L}$ into the Jordan canonical form [44], where it has a block-diagonal structure. This is achieved via the similarity transformation

$$
\mathcal{L}_{J}(t)=S^{-1}(t) \mathcal{L}(t) S(t),
$$

where $\mathcal{L}_{J}(t)=\operatorname{diag}\left(J_{1}, \ldots, J_{m}\right)$ denotes the Jordan form of $\mathcal{L}(t)$. The Jordan blocks $J_{\alpha}$, of dimension $n_{\alpha}$, are always of the form:

$$
J_{\alpha}=\left(\begin{array}{ccccc}
\lambda_{\alpha} & 1 & 0 & \ldots & 0 \\
0 & \lambda_{\alpha} & 1 & \ldots & 0 \\
\vdots & \ddots & \ddots & \ddots & \vdots \\
0 & \ldots & 0 & \lambda_{\alpha} & 1 \\
0 & \ldots & \ldots & 0 & \lambda_{\alpha}
\end{array}\right)
$$

To each Jordan block are associated a left and a right eigenvector with eigenvalue $\lambda_{\alpha}$, which can in general be complex. The number $m$ of Jordan blocks is given by the number of linearly independent eigenstates of $\mathcal{L}(t)$, with each eigenstate associated to a different block $J_{\alpha}$. Since $\mathcal{L}(t)$ is non-Hermitian, we generally do not have a basis of eigenstates, whence some care is required in order to find a basis for describing the density operator. It can be shown [24] that instantaneous right $\left.\left\{\left|\mathcal{D}_{\beta}^{(j)}(t)\right\rangle\right\rangle\right\}$ and left $\left\{\left\langle\left\langle\mathcal{E}_{\alpha}^{(i)}(t)\right|\right\}\right.$ bases in the state space of linear operators can always be systematically constructed, with the following suitable features:

- Orthonormality condition:

$$
\left\langle\left\langle\mathcal{E}_{\alpha}^{(i)}(t) \mid \mathcal{D}_{\beta}^{(j)}(t)\right\rangle\right\rangle={ }_{J}\left\langle\left\langle\mathcal{E}_{\alpha}^{(i)} \mid \mathcal{D}_{\beta}^{(j)}\right\rangle\right\rangle_{J}=\delta_{\alpha \beta} \delta^{i j} .
$$

- Invariance of the Jordan blocks under the action of the Lindblad super-operator:

$$
\begin{aligned}
& \left.\left.\left.\mathcal{L}(t)\left|\mathcal{D}_{\alpha}^{(j)}(t)\right\rangle\right\rangle=\left|\mathcal{D}_{\alpha}^{(j-1)}(t)\right\rangle\right\rangle+\lambda_{\alpha}(t)\left|\mathcal{D}_{\alpha}^{(j)}(t)\right\rangle\right\rangle . \\
& \left\langle\left\langle\mathcal{E}_{\alpha}^{(i)}(t)\right| \mathcal{L}(t)=\left\langle\left\langle\mathcal{E}_{\alpha}^{(i+1)}(t)\right|+\left\langle\left\langle\mathcal{E}_{\alpha}^{(i)}(t)\right| \lambda_{\alpha}(t) .\right.\right.\right.
\end{aligned}
$$

with $\left.\left|\mathcal{D}_{\alpha}^{(-1)}\right\rangle\right\rangle \equiv 0$ and $\left\langle\left\langle\mathcal{E}_{\alpha}^{\left(n_{\alpha}\right)}\right| \equiv 0\right.$. Here the subscripts enumerate Jordan blocks $(\alpha \in\{1, \ldots, m\})$, while the superscripts enumerate basis states inside a given Jordan block $\left(i, j \in\left\{0, \ldots, n_{\alpha}-1\right\}\right)$. 


\section{B. Adiabatic conditions for open quantum system}

Before stating explicitly the conditions for adiabatic evolution, we provide a formal definition of adiabaticity for the case of open systems:

Definition III.1 An open quantum system is said to undergo adiabatic dynamics if its Hilbert-Schmidt space can be decomposed into decoupled Lindblad-Jordan-eigenspaces with distinct, time-continuous, and non-crossing instantaneous eigenvalues of $\mathcal{L}(t)$.

This definition is a natural extension for open systems of the idea of adiabatic behavior. Indeed, in this case the master equation (32) can be decomposed into sectors with different and separately evolving Lindblad-Jordan eigenvalues. The more familiar notion of closed-system adiabaticity is obtained as a special case when the Lindblad superoperator is Hermitian: in that case it can be diagonalized and the Jordan blocks all become one-dimensional, with corresponding real eigenvalues (that correspond to energy differences). The splitting into Jordan blocks of the Lindblad superoperator is achieved through the choice of a basis which preserves the Jordan block structure as, for example, the sets of right $\left.\left\{\left|\mathcal{D}_{\beta}^{(j)}(t)\right\rangle\right\rangle\right\}$ and left $\left\{\left\langle\left\langle\mathcal{E}_{\alpha}^{(i)}(t)\right|\right\}\right.$ vectors introduced above. Such a basis generalizes the notion of Schrödinger-eigenvectors. Based on this concept of adiabaticity, we state below (without the proofs) several theorems which have been derived in Ref. [24].

Theorem III.2 A sufficient condition for open quantum system adiabatic dynamics as given in Definition III.1 is:

$$
\max _{0 \leq s \leq 1}\left|\sum_{p=1}^{\left(n_{\alpha}-i\right)}\left(\prod_{q=1}^{p} \sum_{k_{q}=0}^{\left(j-S_{q-1}\right)}\right) \frac{\left\langle\left\langle\mathcal{E}_{\alpha}^{(i+p-1)}\left|\frac{d \mathcal{L}}{d s}\right| \mathcal{D}_{\beta}^{\left(j-S_{p}\right)}\right\rangle\right\rangle}{(-1)^{S_{p}} \omega_{\beta \alpha}^{p+S_{p}}}\right| \ll 1,
$$

where $s=t / T$ is the scaled time and

$$
\omega_{\beta \alpha}(t)=\lambda_{\beta}(t)-\lambda_{\alpha}(t), \quad S_{q}=\sum_{s=1}^{q} k_{s} \quad\left(\text { with } S_{0}=0\right), \quad\left(\prod_{q=1}^{p} \sum_{k_{q}=0}^{\left(j-S_{q-1}\right)}\right) \equiv \sum_{k_{1}=0}^{j-S_{0}} \ldots \sum_{k_{p}=0}^{j-S_{p-1}}
$$

and where $\lambda_{\beta} \neq \lambda_{\alpha}$, with $i$ and $j$ denoting arbitrary indices associated to the Jordan blocks $\alpha$ and $\beta$, respectively.

The role of the energy differences that appear in the equations for the closed case is played here by the (in general complex-valued) difference between Jordan eigenvalues $\omega_{\beta \alpha}$, while the norm of the time-derivative of the Hamiltonian is replaced here by the norm of $\frac{d \mathcal{L}}{d s}$, evaluated over and inside Jordan blocks. Eq. (42) gives a means to estimate the accuracy of the adiabatic approximation via the computation of the time derivative of the Lindblad superoperator acting on right and left vectors. The norm used in Eq. (42) can be simplified by considering only the term with maximum absolute value, which results in:

Corollary III.3 A sufficient condition for open quantum system adiabatic dynamics is

$$
\mathcal{N}_{i j}^{n_{\alpha} n_{\beta}} \max _{0 \leq s \leq 1}\left|\frac{\left\langle\left\langle\mathcal{E}_{\alpha}^{(i+p-1)}\left|\frac{d \mathcal{L}}{d s}\right| \mathcal{D}_{\beta}^{\left(j-S_{p}\right)}\right\rangle\right\rangle}{\omega_{\beta \alpha}^{p+S_{p}}}\right| \ll 1
$$

where the $\max$ is taken for any $\alpha \neq \beta$, and over all possible values of $i \in\left\{0, \ldots, n_{\alpha}-1\right\}, j \in\left\{0, \ldots, n_{\beta}-1\right\}$, and $p$, with

$$
\mathcal{N}_{i j}^{n_{\alpha} n_{\beta}}=\frac{\left(n_{\alpha}-i+1+j\right) !}{(1+j) !\left(n_{\alpha}-i\right) !}-1
$$

The factor $\mathcal{N}_{i j}^{n_{\alpha} n_{\beta}}$ given in Eq. (45) is just the number of terms of the sums in Eq. (42). We have included a superscript $n_{\beta}$, even though there is no explicit dependence on $n_{\beta}$, since $j \in\left\{0, \ldots, n_{\beta}-1\right\}$.

Furthermore, an adiabatic condition for a slowly varying Lindblad super-operator can directly be obtained from Eq. (42), yielding:

Corollary III.4 A simple sufficient condition for open quantum system adiabatic dynamics is $\dot{\mathcal{L}} \approx 0$. 
Note that this condition is in a sense too strong, since it need not be the case that $\dot{\mathcal{L}}$ is small in general (i.e., for all its matrix elements).

Just as in the closed-systems case, one can also express the condition for adiabaticity in terms of the total time of evolution. To this end, we expand the density matrix for an arbitrary time $t$ in the instantaneous right eigenbasis $\left.\left\{\left|\mathcal{D}_{\beta}^{(j)}(t)\right\rangle\right\rangle\right\}$ as

$$
\left.|\rho(t)\rangle\rangle=\frac{1}{2} \sum_{\beta=1}^{m} \sum_{j=0}^{n_{\beta}-1} p_{\beta}^{(j)}(t) e^{\int_{0}^{t} \lambda_{\beta}\left(t^{\prime}\right) d t^{\prime}}\left|\mathcal{D}_{\beta}^{(j)}(t)\right\rangle\right\rangle
$$

where $m$ is the number of Jordan blocks and $n_{\beta}$ is the dimension of the block $J_{\beta}$. We emphasize that we are assuming that there are no eigenvalue crossings in the spectrum of the Lindblad superoperator during the evolution. We also define

$$
V_{\beta \alpha}^{(i j p)}(s)=p_{\beta}^{(j)}(s)\left\langle\left\langle\mathcal{E}_{\alpha}^{(i+p-1)}(s)\left|\frac{d \mathcal{L}(s)}{d s}\right| \mathcal{D}_{\beta}^{\left(j-S_{p}\right)}(s)\right\rangle\right\rangle
$$

and

$$
\Omega_{\beta \alpha}(t)=\int_{0}^{t} \omega_{\beta \alpha}\left(t^{\prime}\right) d t^{\prime}
$$

Then the following adiabatic time condition can be established [24]:

Theorem III.5 Consider an open quantum system governed by a Lindblad superoperator $\mathcal{L}(s)$. Then the adiabatic dynamics in the interval $0 \leq s \leq 1$ occurs if and only if the following time conditions, obtained for each coefficient $p_{\alpha}^{(i)}(s)$, are satisfied:

$$
T \gg \max _{0 \leq s \leq 1}\left|\sum_{\beta \mid \lambda_{\beta} \neq \lambda_{\alpha}} \sum_{j, p}(-1)^{S_{p}}\left[\frac{V_{\beta \alpha}^{(i j p)}(0)}{\omega_{\beta \alpha}^{p+S_{p}+1}(0)}-\frac{V_{\beta \alpha}^{(i j p)}(s) e^{T \Omega_{\beta \alpha}(s)}}{\omega_{\beta \alpha}^{p+S_{p}+1}(s)}+\int_{0}^{s} d s^{\prime} e^{T \Omega_{\beta \alpha}\left(s^{\prime}\right)} \frac{d}{d s^{\prime}} \frac{V_{\beta \alpha}^{(i j p)}\left(s^{\prime}\right)}{\omega_{\beta \alpha}^{p+S_{p}+1}\left(s^{\prime}\right)}\right]\right|
$$

Theorem 11.5 provides a very general condition for adiabaticity in open quantum systems. Equation (49) simplifies in a number of situations.

- Adiabaticity is guaranteed whenever $V_{\beta \alpha}^{(i j p)}(s)$ vanishes for all $\lambda_{\alpha} \neq \lambda_{\beta}$.

- Adiabaticity is similarly guaranteed whenever $V_{\beta \alpha}^{(i j p)}(s)$, which can depend on $T$ through $p_{\beta}^{(j)}$, vanishes for all $\lambda_{\alpha}, \lambda_{\beta}$ such that $\operatorname{Re}\left(\Omega_{\beta \alpha}\right)>0$ and does not grow faster, as a function of $T$, than $\exp \left(T\left|\operatorname{Re} \Omega_{\beta \alpha}\right|\right)$ for all $\lambda_{\alpha}, \lambda_{\beta}$ such that $\operatorname{Re}\left(\Omega_{\beta \alpha}\right)<0$.

- When $\operatorname{Re}\left(\Omega_{\beta \alpha}\right)=0$ and $\operatorname{Im}\left(\Omega_{\beta \alpha}\right) \neq 0$ the integral in inequality (49) vanishes in the infinite time limit due to the Riemann-Lebesgue lemma [33], as in the closed case discussed before. In this case, again, adiabaticity is guaranteed provided $p_{\beta}^{(j)}(s)$ [and hence $V_{\beta \alpha}^{(i j p)}(s)$ ] does not diverge as a function of $T$ in the limit $T \rightarrow \infty$.

- When $\operatorname{Re}\left(\Omega_{\beta \alpha}\right)>0$, the adiabatic regime can still be reached for large $T$ provided that $p_{\beta}^{(j)}(s)$ contains a decaying exponential which compensates for the growing exponential due to $\operatorname{Re}\left(\Omega_{\beta \alpha}\right)$.

- Even if there is an overall growing exponential in inequality (49), adiabaticity could take place over a finite time interval $\left[0, T_{*}\right]$ and, afterwards, disappear. In this case, which would be an exclusive feature of open systems, the crossover time $T_{*}$ would be determined by an inequality of the type $T \gg a+b \exp (c T)$, with $c>0$. The coefficients $a, b$ and $c$ are functions of the system-bath interaction. Whether the latter inequality can be solved clearly depends on the values of $a, b, c$, so that a conclusion about adiabaticity in this case is model dependent.

A simpler sufficient condition can be derived from Eq. (49) by considering the term with maximum absolute value in the sum. This procedure leads to the following corollary:

Corollary III.6 A sufficient time condition for the adiabatic regime of an open quantum system governed by a Lindblad superoperator $\mathcal{L}(t)$ is

$$
T \gg \mathcal{M}_{i j}^{n_{\alpha} n_{\beta}} \max _{0 \leq s \leq 1}\left|\frac{V_{\beta \alpha}^{(i j p)}(0)}{\omega_{\beta \alpha}^{p+S_{p}+1}(0)}-\frac{V_{\beta \alpha}^{(i j p)}(s) e^{T \Omega_{\beta \alpha}(s)}}{\omega_{\beta \alpha}^{p+S_{p}+1}(s)}+\int_{0}^{s} d s^{\prime} e^{T \Omega_{\beta \alpha}\left(s^{\prime}\right)} \frac{d}{d s^{\prime}} \frac{V_{\beta \alpha}^{(i j p)}\left(s^{\prime}\right)}{\omega_{\beta \alpha}^{p+S_{p}+1}\left(s^{\prime}\right)}\right|,
$$


where max is taken over all possible values of the indices $\lambda_{\alpha} \neq \lambda_{\beta}, i, j$, and $p$, with

$$
\mathcal{M}_{i j}^{n_{\alpha} n_{\beta}}=\sum_{\beta \mid \lambda_{\beta} \neq \lambda_{\alpha}} \sum_{j=0}^{\left(n_{\beta}-1\right)} \sum_{p=1}^{\left(n_{\alpha}-i\right)}\left(\prod_{q=1}^{p} \sum_{k_{q}=0}^{\left(j-S_{q-1}\right)}\right) 1=\Lambda_{\beta \alpha}\left[\frac{\left(n_{\alpha}+n_{\beta}-i+1\right) !}{\left(n_{\alpha}-i+1\right) ! n_{\beta} !}-n_{\beta}-1,\right]
$$

where $\Lambda_{\beta \alpha}$ denotes the number of Jordan blocks such that $\lambda_{\alpha} \neq \lambda_{\beta}$.

Further discussion of the physical significance of these adiabaticity conditions, as well as an illustrative example, can be found in Ref. 24]. The application of the adiabatic theorem for open quantum systems to problems in quantum information processing (e.g., in the context of adiabatic quantum computing [21, 22, 23]) and geometric phases [45, 46, 47], seems particularly appealing as a venue for future research.

\section{THE MARZLIN-SANDERS INCONSISTENCY}

The adiabatic theorem can be deceptively simple when it is not carefully interpreted. In a recent paper entitled K.-P. Marzlin and B.C. Sanders argue that there may be an inconsistency in the adiabatic theorem for closed quantum systems [26], when the change in instantaneous adiabatic eigenstates is significant. Here we simplify their argument and show where exactly is the fallacy that leads one to conclude that there is such an inconsistency.

\section{A. The condition for adiabaticity revisited}

Let us consider a quantum system evolving unitarily under the Schrödinger equation (1). At the initial time $t_{0}$ the system is assumed to be in the particular instantaneous energy eigenstate $\left|E_{0}\left(t_{0}\right)\right\rangle$. For a general time $t$ the evolution of the system is described by

$$
|\psi(t)\rangle=U\left(t, t_{0}\right)\left|E_{0}\left(t_{0}\right)\right\rangle
$$

where $U\left(t, t_{0}\right)=\mathcal{T} \exp \left(-i \int_{t_{0}}^{t} H\left(t^{\prime}\right) d t^{\prime}\right)$ is the unitary evolution operator. Assuming that the Hamiltonian changes slowly in time and that $\left|E_{0}\left(t_{0}\right)\right\rangle$ is non-degenerate, the adiabatic theorem implies that

$$
|\psi(t)\rangle=e^{-i \int^{t} E_{0}} e^{i \beta_{0}}\left|E_{0}(t)\right\rangle
$$

where $\int^{t} E_{0} \equiv \int_{t_{0}}^{t} E_{0}\left(t^{\prime}\right) d t^{\prime}$ and the Berry's phase $\beta_{0}$ is given by $\beta_{0}=i \int\left\langle E_{0} \mid \dot{E}_{0}\right\rangle$. Therefore the substitution of Eq. (531) into the instantaneous eigenbasis of $H(t)$, defined by $H(t)\left|E_{n}(t)\right\rangle=E_{n}(t)\left|E_{n}(t)\right\rangle$, yields

$$
H(t)|\psi(t)\rangle=E_{0}(t)|\psi(t)\rangle,
$$

which simply states that the wave function is an instantaneous eigenstate of the Hamiltonian in the adiabatic regime. Substituting Eq. (54) into the Schrödinger equation (1) one obtains

$$
i|\dot{\psi}\rangle=E_{0}(t)|\psi(t)\rangle .
$$

It is important to observe that the above equation has been derived by using the fact that the adiabatic solution must be an instantaneous eigenstate of the Hamiltonian. Moreover one can see that the adiabatic wave function is really a solution of the adiabatic Schrödinger equation by substituting Eq. (53) into Eq. (55), from which one obtains

$$
\left(1-\left|E_{0}(t)\right\rangle\left\langle E_{0}(t)\right|\right)\left|\dot{E}_{0}(t)\right\rangle=0 .
$$

In order to show that Eq. (56) is obeyed in the adiabatic regime we can project this equation by multiplying it by each instantaneous basis vector $\left\langle E_{n}(t)\right|$ :

$$
\left\langle E_{n}(t)\left|\left(1-\left|E_{0}(t)\right\rangle\left\langle E_{0}(t)\right|\right)\right| \dot{E}_{0}(t)\right\rangle=0 .
$$

Therefore we obtain that the above equation is satisfied, for each $n$, if the adiabatic constraints [see Eq. (7)] are obeyed

$$
\left|\frac{\left\langle E_{n}(t) \mid \dot{E}_{0}(t)\right\rangle}{E_{0}(t)-E_{n}(t)}\right| \ll 1, \quad(n \neq 0)
$$




\section{B. The inconsistent step}

Now suppose that we wish to solve the adiabatic Schrödinger equation (55), but (incorrectly) ignore the fact that it has been derived by assuming that $|\psi(t)\rangle$ is an instantaneous eigenstate of $H(t)$. Then, imposing the initial condition $\left|\psi\left(t_{0}\right)\right\rangle=\left|E_{0}\left(t_{0}\right)\right\rangle$, one easily finds that Eq. (55) is satisfied by the following wave function:

$$
|\psi(t)\rangle=e^{-i \int^{t} E_{0}}\left|E_{0}\left(t_{0}\right)\right\rangle .
$$

However, this $|\psi(t)\rangle$ is clearly inconsistent with Eq. (54) and therefore is an illegal solution, since it generally is not an instantaneous eigenstate of the Hamiltonian. Indeed, the general adiabatic solution is given by Eq. (53), which includes the Berry's phase and $\left|E_{0}(t)\right\rangle$, as opposed to $\left|E_{0}\left(t_{0}\right)\right\rangle$. In fact, if we take Eq. (59) as the adiabatic wave function and substitute it into Eq. (53) we obtain

$$
e^{i \beta_{0}}\left|E_{0}(t)\right\rangle=\left|E_{0}\left(t_{0}\right)\right\rangle .
$$

Then multiplying Eq. (60) by $\left\langle E_{0}\left(t_{0}\right)\right|$

$$
e^{i \beta_{0}}\left\langle E_{0}\left(t_{0}\right) \mid E_{0}(t)\right\rangle=1 .
$$

This inconsistency is precisely the one claimed by Marzlin and Sanders in Ref. [26], Eq. (6). Note that this result is obtained there in a somewhat more complicated manner, by considering the adiabatic solution of a time-reversed wave function $|\bar{\psi}(t)\rangle=U^{\dagger}\left(t, t_{0}\right)\left|E_{0}\left(t_{0}\right)\right\rangle$. We note that the solution for $|\bar{\psi}(t)\rangle$ [their Eq. (4)] is very similar to our Eq. (59). Hence, in the same way that we have been led to an inconsistent result due to a deliberately wrong adiabatic solution for $|\psi(t)\rangle$, Ref. [26] has been led to an inconsistent solution for their $|\bar{\psi}(t)\rangle$.

\section{CONCLUSION}

We have reviewed the adiabatic dynamics of both closed and open quantum systems. In the case of closed systems the adiabatic limit is the case where initial Schrödinger-eigenspaces evolve independently, without any transitions between eigenspaces; this limit can be relaxed and a perturbation theory can be developed in the number of transitions. In the case of open systems the notion of Schrödinger-eigenspaces is replaced by independently evolving Lindblad-Jordan blocks. A corresponding perturbation theory has not yet been developed. We have also shown that the inconsistency in the adiabatic theorem claimed in Ref. [26] is a consequence of an improper adiabatic solution for the wave function. One arrives at an inconsistent result by taking the instantaneous adiabatic eigenstates and integrating them over all time using the adiabatic Schrödinger equation. The adiabatic theorem remains a valuable and consistent tool for studying the dynamics of slowly evolving quantum systems.

\section{Acknowledgments}

The research of the authors is sponsored by CNPq-Brazil (to M.S.S.), and the Sloan Foundation, PREA and NSERC (to D.A.L.).

[1] P. Ehrenfest, Ann. d. Phys. 51, 327 (1916).

[2] M. Born and V. Fock, Zeit. f. Physik 51, 165 (1928).

[3] T. Kato, J. Phys. Soc. Jap. 5, 435 (1950).

[4] A. Messiah, Quantum mechanics (North-Holland, Amsterdam, 1962), Vol. 2.

[5] M.V. Berry, Proc. R. Soc. London A 414, 31 (1987).

[6] N. Nakagawa, Ann. Phys. 179, 145 (1987).

[7] C.P. Sun, J. Phys. A 21, 1595 (1988).

[8] Z. Wu, Phys. Rev. A 40, 2184 (1989).

[9] L.D. Landau, Zeitschrift 2, 46 (1932).

[10] C. Zener, Proc. Roy. Soc. London Ser. A 137, 696 (1932).

[11] M. Gell-Mann and F. Low, Phys. Rev. 84, 350 (1951).

[12] M.V. Berry, Proc. Roy. Soc. (Lond.) 392, 45 (1989).

[13] F. Wilczek and A. Zee, Phys. Rev. Lett. 52, 2111 (1984). 
[14] P. Zanardi and M. Rasetti, Phys. Lett. A 264, 94 (1999).

[15] J. Pachos, P. Zanardi, and M. Rasetti, Phys. Rev. A 61, 010305 (2000).

[16] J.A. Jones, V. Vedral, A. Ekert, and G. Castagnoli, Nature 403, 869 (2000).

[17] J. Pachos and S. Chountasis, Phys. Rev. A 62, 052318 (2000).

[18] L.-M. Duan, J. I. Cirac, and P. Zoller, Science 292, 1695 (2001).

[19] I. Fuentes-Guridi, J. Pachos, S. Bose, V. Vedral, and S. Choi, Phys. Rev. A 66, 022102 (2002).

[20] L. Faoro, J. Siewert, and R. Fazio, Phys. Rev. Lett. 90, 028301 (2003).

[21] E. Farhi, J. Goldstone, S. Gutmann, and M. Sipser, LANL Preprint quant-ph/0001106

[22] E. Farhi, J. Goldstone, S. Gutmann, J. Lapan, A. Lundgren, and D. Preda, Science 292, 472 (2001).

[23] D. Aharonov, W. v. Dam, J. Kempe, Z. Landau, S. Lloyd, O. Regev, LANL Preprint quant-ph/0405098

[24] M.S. Sarandy and D.A. Lidar, LANL Preprint quant-ph/0404147. Phys. Rev. A (2004), in press.

[25] D.A. Lidar and K.B. Whaley, in Irreversible Quantum Dynamics, Vol. 622 of Lecture Notes in Physics, edited by F. Benatti and R. Floreanini (Springer, Berlin, 2003), p. 83, LANL Preprint quant-ph/0301032

[26] K.-P. Marzlin and B.C. Sanders, Phys. Rev. Lett. 93, 160408 (2004).

[27] D.M. Tong, K. Singh, L.C. Kwek, and C.H. Oh, LANL Preprint quant-ph/0406163 (2004).

[28] A.K. Pati and A.K. Rajagopal, LANL Preprint quant-ph/0405129 (2004).

[29] Z. Wu and H. Yang, LANL Preprint quant-ph/0410118 (2004).

[30] A. Mostafazadeh, Dynamical Invariants, Adiabatic Approximation, and the Geometric Phase (Nova Science Publishers, New York, 2001).

[31] J.E. Avron and A. Elgart, Phys. Rev. A 58, 4300 (1998); Commun. Math. Phys. 203, 445 (1999).

[32] K. Gottfried and T.-M. Yan, Quantum Mechanics: Fundamentals (Springer, New York, 2003).

[33] J.W. Brown and R.V. Churchill, Fourier series and boundary value problems (McGraw-Hill, New York, 1993).

[34] The Riemann-Lebesgue lemma can be stated through the proposition: Let $f:[a, b] \rightarrow \mathbf{C}$ be an integrable function on the interval $[a, b]$. Then $\int_{a}^{b} d x e^{i n x} f(x) \rightarrow 0$ as $n \rightarrow \pm \infty$.

[35] L.-B. Fu, J.-L. Chen, J. Phys. A 37, 3699 (2004); E. Sjöqvist, LANL Preprint quant-ph/0404174 K.-P. Marzlin, S. Ghose, B.C. Sanders, LANL Preprint quant-ph/0405052 D.M. Tong, E. Sjöqvist, L.C. Kwek, C.H. Oh, LANL Preprint quant-ph/0405092 R.S. Whitney, Y. Makhlin, A. Shnirman, Y. Gefen, LANL Preprint cond-mat/0405267 I. Kamleitner, J.D. Cresser, B.C. Sanders, LANL Preprint quant-ph/0406018

[36] H.-P. Breuer and F. Petruccione, The Theory of Open Quantum Systems (Oxford University Press, Oxford, 2002).

[37] K. Kraus, Ann. of Phys. 64, 311 (1971).

[38] R. Alicki and K. Lendi, Quantum Dynamical Semigroups and Applications, No. 286 in Lecture Notes in Physics (SpringerVerlag, Berlin, 1987).

[39] V. Gorini, A. Kossakowski, and E.C.G Sudarshan, J. Math. Phys. 17, 821 (1976).

[40] G. Lindblad, Commun. Math. Phys. 48, 119 (1976).

[41] D.A. Lidar, Z. Bihary, and K.B. Whaley, Chem. Phys. 268, 35 (2001).

[42] K. Lendi, Phys. Rev. A 33, 3358 (1986).

[43] H.-P. Breuer, Phys. Rev. A 70, 012106 (2004).

[44] R.A. Horn and C.R. Johnson, Matrix Analysis (Cambridge University Press, Cambridge, UK, 1999).

[45] A.C.A. Pinto and M.T. Thomaz, J. Phys. A 36, 7461 (2003).

[46] A. Carollo, I. Fuentes-Guridi, M.F. Santos, and V. Vedral, Phys. Rev. Lett. 92, 020402 (2004).

[47] I. Kamleitner, J.D. Cresser, and B.C. Sanders, e-print quant-ph/0406018 (2004). 